\title{
Noma-Stbcfor Uplink Mimo Channel
}

\author{
K. Kavitha, N. M. Harshini
}

\begin{abstract}
This paper bringforths the concept of space time block codes for Non-Orthogonal Multiple Access (NOMA). Wireless communication networks are rapidly driven to meet the requirements interms of spectral/power efficiency. NOMA serves as promising technology in meeting these requirements by means of breaking orthogonality and serving all the users in same time/frequency domain by only differing in their power levels. In this paper, NOMA is combained with MIMO signal processing technique is proposed to improve the quality of the received signal. This paper compares the Bit Error Performance (BER) of two user NOMA system in existence of STBC and in devoid of STBC over Rayleigh fading channel. The simulation results show that the proposed system could give improved BER performance for the data stream multiplexed along with the main stream data even with imperfect Channel State Information.
\end{abstract}

Keywords- Space Time Codes, Non-Orthogonal Multiple Access, STBC, SIC, MIMO..

\section{INTRODUCTION}

In recent trends, NOMA has been an innovation in promising the multiplexing technique in power domain for multiple users to share the same time and frequency resource [1]. To respond the mobile traffic in forthcoming decades improvement in system capacity and Quality of Users has to be required[2]. As a novel aproach, NOMA method was implemented where several users based on the different channel conditions are multiplexed at transmitter and signals of multiple users are separated at receiver. NOMA is classified into power domain and code domain multiplexing. Power domain NOMA uses Multi User Superposition Transmission (MUST) most promising technique for improving the spectral efficiency [3]. In NOMA, multiple users are shares the spectrum simultaneously and [4] decoded using Successive Interference Cancellation (SIC) receiver at receiver [7]. Massive connctivity, low latency, high spectral efficiency are the major advantages that drives NOMA to be more potential. NOMA in uplink scenario [8] is shown in Figure.1. NOMA serves various users with same degrees of freedom where they are spitted by means of power domain. Since SIC is employed at receiver, unequal rates are achieved by users and this serves a major problem in terms of strict fairness conditions. The data of weak users can be relayed by means of strong users but extra channel resources are needed for this and this serves as a technique for improving fairness. Power allocation can also support fairness improvement [9].Since, the presence of the other un-decoded user data streams are considered as a part of additive noise, the BER performance of the NOMA system would be inferior compared to single user system. In recent

Revised Manuscript Received on 14 August, 2019.

Dr. K.Kavitha, Professor, Department of ECE,Kumaraguru College of Technology, Coimbatore, Tamilnadu, India.(Email kavitha.k.ece@kct.ac.in)

N.M. Harshini, PG Scholar, Department of ECE,Kumaraguru College of Technology, Coimbatore, Tamilnadu, India years, MIMO becomes the inherent part of the wireless communication systems and standards. Signal processing for MIMO is a vast explored area in the past two decades due to its advantages $[12,13,14,15]$.Space Time Coding (STC) are the diversity maximization techniques which would achieve improved performance over wireless channel. STC techniques are broadly classified into Space Time Block Codes (STBC) and Space Time Trellis Codes (STTC)[10,11]. In the paper [16], the power controlled STBC NOMA system has been explored over MIMO channel meant for downlink scenario. In this paper multi antenna system with simple Space Time Block coding technique is proposed to improve the performance of the NOMA system over wireless channel meant for uplink. The organization of the paper is given as below: In the section II the system model of the proposed NOMA-STBC system is elaborated. Section III briefs about the simulation results and analysis and section IV gives the paper summary.

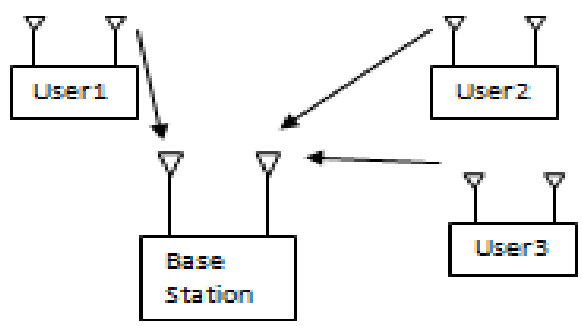

Figure 1 Uplink NOMA

\section{SYSTEM MODEL}

Consider a uplink scenario with single cell withN number of users with in presence of single base station where users are equipped with multi antenna in quasi static and identically distributed fading channels. Each base station transmits and receives data from all the users with respect to power constraint $\mathrm{P}$ as described in [7]. Each channel is sorted in accordance with weakest channel as follows

$$
\mathbf{0}<\left|\boldsymbol{h}_{1}\right|^{2} \leq\left|\boldsymbol{h}_{2}\right|^{2} \leq \cdots \leq\left|\boldsymbol{h}_{i}\right|^{2} \ldots \leq\left|\boldsymbol{h}_{N}\right|^{2}
$$

Where $U_{i}$ user always holds weakest channel[3].

NOMA serves all the users with its entire bandwidth at transmitter and at the receiver using SIC simultaneously. The power domain is accomplished by means of user multiplexing.

The data stream to be transmitted at the user is encoded by channel encoder and STBC encoders which maps the binary data into equivalent complex values of two user signals at transmitter. Two dimensional modulation technique such as Quadrature Phase Shift Keying (QPSK) is retained here. At transmitter, the multiple user's information 
are transmitted simultaneously over MIMO channel. The transmitted signal by $\mathrm{n}^{\text {th }}$ user is given by $\boldsymbol{S}_{n}$, Where, $S_{n}=\left[s_{n 1}, s_{n 2}, s_{n 3} \ldots \ldots . s_{n_{N_{t}}}\right]^{T}$ is the STBC encoded data of size $\left(N_{t} \times 1\right)[10]$. The $\mathrm{N}$ user data are received by the base station simultaneously from $\mathrm{N}$ different transmitters.

$$
\begin{aligned}
\boldsymbol{y} & =\sum_{\boldsymbol{n}=\mathbf{1}}^{\boldsymbol{N}} \mathcal{H}_{\boldsymbol{n}} \times \boldsymbol{S}_{\boldsymbol{n}}+\mathcal{W}(1) \\
\boldsymbol{H}_{\boldsymbol{n}} & =\left[\begin{array}{cccc}
h_{11}^{n} & h_{12}^{n} & \cdots & h_{1 N_{t}}^{n} \\
h_{21}^{n} & h_{21}^{n} & \cdots & h_{2 N_{t}}^{n} \\
\vdots & \cdots & \cdots & \vdots \\
h_{N_{r} 1}^{n} & h_{N_{r} 1}^{n} & \cdots & h_{N_{r} N_{t}}^{n}
\end{array}\right]
\end{aligned}
$$

Where, $\boldsymbol{H}_{\boldsymbol{n}}$ is the MIMO channel matrix and $h_{i j}^{n}$ is the iid Rayleigh fading channel co-efficient of the path linking $n_{r}{ }^{t h}$ receiver antenna and $n_{t}{ }^{\text {th }}$ transmitter antenna in the $N_{r} \times N_{t}$ MIMO frequency non selective slow fading channel.

$\mathcal{W}=\left[w_{1}, w_{2} \ldots w_{N_{r}}\right]^{T}$, where, $w_{n_{r}}$ is AWGN (Additive White Gaussian Noise) noise added with zero mean and variance $N_{0} / 2$ to the signal received through $n_{r}{ }^{\text {th }}$ antenna at the receiver.

The receiver is believed to hold the channel state information (CSI). At the receiver SIC technique is used to detect the signal as given in the Figure 3 . The strong signal is decoded first by considering the other user signals as a part of noise. The detected data stream of the first user is used to regenerate the received user data of the first user and subtracted from the noisy received signal which would reduce the multiple access interference (MAI) at the receiver to decode the next strongest data received

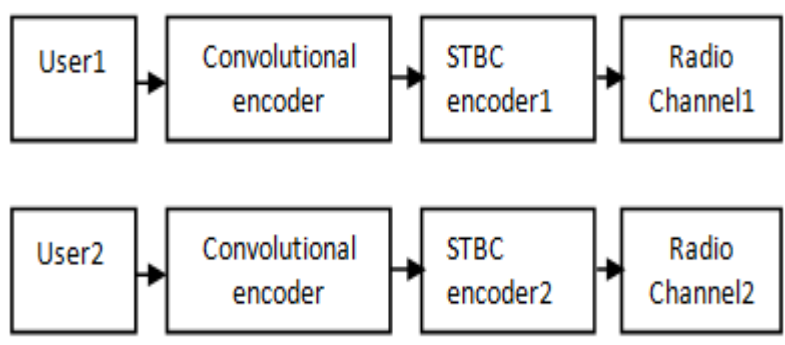

Figure 2 NOMA STBC Transmitter

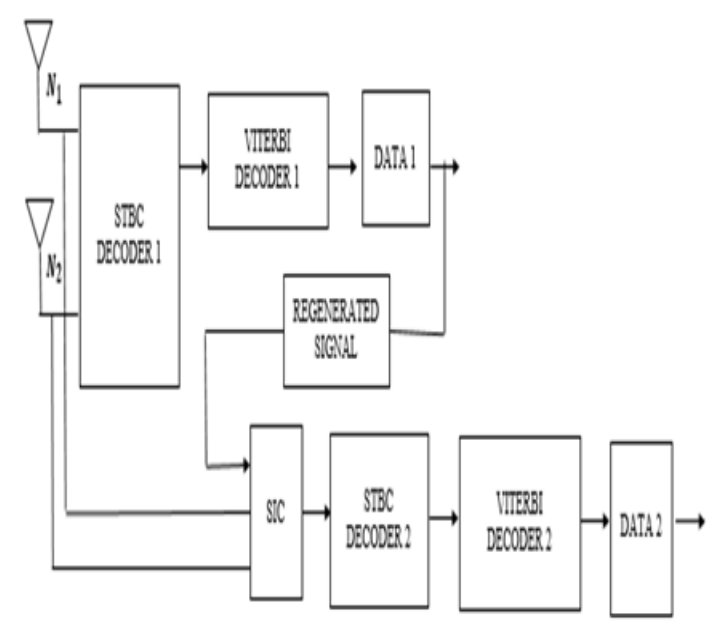

Figure 3 SIC Receiver
For two user scenario the received signal is given by equation (3).

$$
\mathcal{Y}=\mathcal{H}_{1} S_{1}+\mathcal{H}_{2} S_{2}+\mathcal{W}(3)
$$

Where $\boldsymbol{S}_{\mathbf{1}}$ and $\boldsymbol{S}_{\mathbf{2}}$ are thetransmitteruplink user signals. $\mathcal{H}_{\mathbf{1}}$ and $\mathcal{H}_{\mathbf{2}}$ are the corresponding CSI of the received signals at the base station.Average path gains of the received signals are compared to decide which user to be detected first using SIC technique. In first stage by treating weak user as noise, the strong user signal is decoded. In second stage, the decoded strong user signal is regenerated and subtracted from noisy received signal and there after weak user decoded.

\section{RESULTS AND DISCUSSION}

BER performance of two user proposed NOMA-STBC has been compared with NOMA without STBC and STBC with no NOMA over fading channel in the Figure 5. The system model for the simulation of transmitted signal is shown in Figure 2. Simulation is done for two user system. $1 / 2$ rate convolution encoder is used

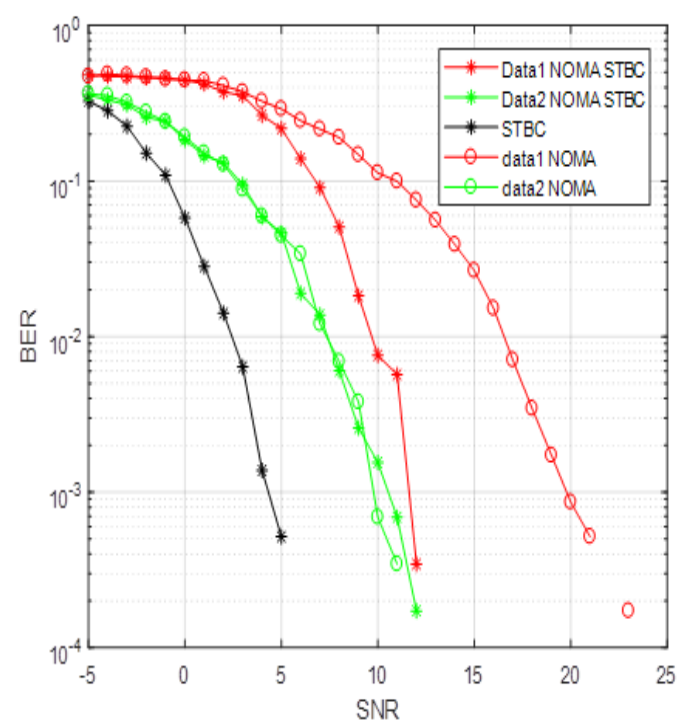

Figure 5BER performance of STBC NOMA

streams transmitted using MOMA. STBC encoder is used to encode these channel for both the data streams and the channel encoded signals given by equation (2). Serial Interference cancellation (SIC) used at the receiver to decode the data. The strong data sequence is decoded first for which data stream 1 acts as interference for data stream2 in the first iteration. The second data stream 2 modulated signal is generated and subtracted from the received noisy signal and the resultant is used to detect the second data stream1. Imperfect Channel State Information (CSI) assumed to be available at the receiver for all the techniques compared in the figure 5. The results show that the use of STBC with NOMA improves the overall BER performance of the system with imperfect channel estimates at the receiver. 


\section{CONCLUSION}

In this paper Multi antenna NOMA-STBC system is proposed to enhance the spectral efficiency suitable for uplink MIMO wireless channel. The proposed system is compared with and without STBC. The simulation results reveal that the use of MIMO system improves the overall BER performance of NOMA with STBC. Further, by increasing the number antenna used in the MIMO channel, it is possible to achieve desirable performance. The simulation is carried out with assumption that only imperfect estimate of the CSI is available at the receiver. From the results it is understood that the perfect knowledge of CSI does not affect the overall performance of the proposed system compared to conventional NOMA.

\section{REFERENCES}

1. Subrat Kumar Sethi, Saswati Paramita, "Network Technology Trend for Next Generation Wireless Communication", National Conference on Recent Advancements in Electronics (NCRAE), 28 January 2016.

2. Yuanwei Liu, Zhijin Qin, Maged Elkashlan, Zhiguo Ding, Arumugam Nallanathan, Lajos Hanzo ,"NonOrthogonal Multiple Access for 5G and Beyond", Proceedings of IEEE, Vol.no. 105, December 2017, pg.no. 2347-2381.

3. S. M. Riazul Islam, Nurilla Avazov, Octavia A. Dobre and Kyung-Sup Kwak, "Power-Domain Non-Orthogonal Multiple Access (NOMA) in 5G Systems: Potentials and Challenges", IEEE communications surveys \& tutorials, vol. 19, no. 2, second quarter 2017.

4. Li Anxin, Lan Yang, Chen Xiaohang, Jiang Huiling, "Non-orthogonal Multiple Access (NOMA) for Future Downlink Radio Access of 5G", China Communications, Supplement No.1 2015, pg.28 -37.

5. Zhiguo Ding, Xianfu Lei, George K. Karagiannidis, Robert Schober, Jinhong Yuan, Vijay K. Bhargava, "A Survey on Non-Orthogonal Multiple Access for 5G Networks: Research Challenges and Future Trends", IEEE journal on selected areas in communications, vol. 35, no.10, October 2017, pp. 2181-2195.

6. S. M. Riazul Islam, Nurilla Avazov, Octavia A. Dobre and Kyung-Sup Kwak, "Power-Domain Non-Orthogonal Multiple Access (NOMA) in 5G Systems: Potentials and Challenges", IEEE communications surveys \& tutorials, vol. 19, no. 2, second quarter 2017.

7. Shun Tomida, Kenichi Higuchi, "Non-orthogonal access with SIC in cellular downlink for user fairness enhancement", Intelligent Signal Processing and Communications Systems (ISPACS),06 February 2012.

8. Yuya Saito, Anass Benjebbour, Yoshihisa Kishiyama, and Takehiro Nakamura, "System-Level Performance Evaluation of Downlink Non-orthogonal Multiple Access (NOMA)", 2013 IEEE 24th International Symposium on Personal, Indoor and Mobile Radio Communications: Fundamentals and PHY Track, pp. 611- 615, 25 November 2013.

9. Stelios Timotheou, and Ioannis Krikidis," Fairness for Non-Orthogonal Multiple Access in 5G Systems", IEEE signal processing letters, vol. 22, no. 10, October 2015, pp. $1647-1651$.

10. Alamouti, SM,"A simple transmit diversity technique for wireless communications", IEEE Journal of Selected Areas in Communications, vol. 16, no. 8, pp.1451-1458, 1998.

11. Tarokh, V, Seshadri, N \&Calderbank, AR, "'Space-time codes for high data rate wireless communication: performance analysis and code construction", IEEE
Transactions onInformation Theory, vol. 44, no. 2 pp.744-765.1998.

12. Kavitha, K \& Mangalam, H 2014, Multilevel Spatial Multiplexing -Space Time Trellis Coded Modulation system for fast fading MIMO Channel?, International Journal of Engineering and Technology (IJET), vol. 6, no. 1, pp. 217-222.

13. Kavitha, K \& Mangalam, H (2016), "Low Complexity Decoding Algorithm for Multilevel Space Time Trellis Codes over MIMO channel", International Journal of Information and Communication Technology. (IJICT), vol.8, no.1, pp. 69-78.

14. Kavitha, K \& Mangalam, H 2016, "Multilevel spatial modulation", Journal of the Chinese Institute of Engineers (JCIE), vol. 39, issue: 6, pp. 713-721

15. K. Kavitha, A. Kumaresan and S. Arun Kumar, "Performance Analysis of Multilevel Spatial Modulation OFDM Technique (MLSM-MIMO)", International Journal of Pure and Applied Mathematics, Volume 116 No. 11 2017, 101-109.

16. Harshini N.M and K.Kavitha, "PERFORMANCE ANALYSIS OF STBC-NOMA", International Journal of Pure and Applied Mathematics Volume 118 No. 20 2018, pp. 1205-1209. 